\title{
Primary dumbbell-shaped epidural myxoid liposarcoma of the thoracic spine: A case report and review of the literature
}

\author{
YOICHI KANEUCHI ${ }^{1}$, MICHIYUKI HAKOZAKI ${ }^{1}$, HITOSHI YAMADA ${ }^{1}$, TAKAHIRO TAJINO ${ }^{1,2}$, \\ KAZUYUKI WATANABE ${ }^{1}$, KOJI OTANI ${ }^{1}$, HIROSHI HOJO ${ }^{3}$, TADASHI HASEGAWA ${ }^{4}$ and SHINICHI KONNO ${ }^{1}$ \\ ${ }^{1}$ Department of Orthopaedic Surgery, Fukushima Medical University School of Medicine, Fukushima 960-1295; \\ ${ }^{2}$ Division of Orthopaedic Surgery, Southern Tohoku Fukushima Hospital, Fukushima 960-2102; \\ ${ }^{3}$ Department of Diagnostic Pathology, Aizu Medical Center, Fukushima Medical University, Fukushima 969-3492; \\ ${ }^{4}$ Department of Surgical Pathology, Sapporo Medical University School of Medicine, Hokkaido 060-8453, Japan
}

Received November 25, 2014; Accepted October 21, 2015

DOI: $10.3892 / \mathrm{ol} .2016 .4089$

\begin{abstract}
Myxoid liposarcoma frequently occurs in the deep soft tissue of the extremities, particularly in the thigh. The present study describes an extremely rare case of primary dumbbell-shaped epidural myxoid liposarcoma of the thoracic spine occurring in a 22-year-old woman. Although preoperative magnetic resonance imaging findings were thought to be compatible with schwannoma, the pathological diagnosis of the resected tumor was myxoid liposarcoma. The patient underwent three courses of adjuvant chemotherapy with doxorubicin and ifosfamide, and exhibited no symptoms or signs of local recurrence or metastasis for 18 months post surgery. The present case suggests that, when radiologically diagnosing spinal epidural tumors, clinicians should consider the possibility of myxoid liposarcoma.
\end{abstract}

\section{Introduction}

Myxoid liposarcoma (MLS) accounts for $~ 5 \%$ of all soft tissue sarcomas and $15-20 \%$ of liposarcomas in adults (1). This second most common subtype of liposarcoma usually occurs in the soft tissue of the extremities; the thigh is affected in more than two-thirds of cases (1-3). MLS is a disease of young adults and is equally common in males and females (1). Liposarcoma originates from primitive mesenchymal cells rather than from mature adipose cells $(2,3)$. Usually, symptoms develop slowly as a painless mass within the deep soft tissue $(1,4)$. One third of the MLS patients develop distant metastasis, particularly

Correspondence to: Dr Michiyuki Hakozaki, Department of Orthopaedic Surgery, Fukushima Medical University School of Medicine, 1 Hikarigaoka, Fukushima-shi, Fukushima 960-1295, Japan

E-mail: paco@fmu.ac.jp

Key words: adjuvant chemotherapy, dumbbell tumor, magnetic resonance imaging, myxoid liposarcoma, spinal epidural tumor to extrapulmonary location, including the retroperitoneum, opposite extremity, axilla and bone $(1,5,6)$.

The current study reports an extremely rare case of primary dumbbell-shaped epidural MLS of the thoracic spine, which radiologically mimicked a schwannoma. Written informed consent was obtained from the patient prior to publication of this case report.

\section{Case report}

A previously healthy 22-year-old woman presented with a one-month history of back pain, hypalgesia and hypesthesia below the level of the xiphoid cartilage, and numbness of the lower extremities. Laboratory findings indicated no abnormalities; however, a neurological examination revealed muscular weakness and increased deep tendon reflexes in the bilateral lower extremities.

Plain radiographs and computed tomography (CT) of the spine indicated no abnormality. However, magnetic resonance imaging (MRI) revealed an epidural tumor progressing in the right foramen of the 5th and 6th thoracic vertebrae, measuring $4.6 \times 3.7 \times 1.4 \mathrm{~cm}$, and exhibiting low intensity on T1-weighted imaging (T1-WI) (Fig. 1A), high intensity on T2-weighted imaging (T2-WI) (Fig. 1B), and diffuse enhancement on gadolinium-enhanced T1-weighted fat-suppression imaging (Fig. 1C). These findings were compatible with schwannoma. As the paralysis of the patient's lower extremities rapidly progressed and gait disturbance appeared, surgical treatment was performed. Laminectomy of the 4th, 5th and 6th thoracic vertebrae was conducted, and the whitish myxoid tumor was subsequently resected piece by piece. Following the surgery, the patient's paralysis recovered rapidly without any disturbance.

Microscopic evaluation of the tumor revealed proliferation of uniform, mononuclear, short spindle- or oval-shaped cells arranged in a multi-nodular pattern (Fig. 2A-C). A growth pattern similar to that of pulmonary edema was also observed (1). Immunohistochemical staining indicated that the tumor cells were positive for vimentin and S-100 protein (Fig. 2D), but negative for mouse double minute 2 homolog and cyclin-dependent kinase 4. A 'chicken-wire' pattern 
Table I. Summary of previously reported cases of spinal epidural myxoid liposarcoma.

\begin{tabular}{lcccccc}
\hline Author (reference no.) & $\begin{array}{c}\text { Age } \\
\text { (years) }\end{array}$ & Gender & Site & $\begin{array}{c}\text { Primary/ } \\
\text { metastasis }\end{array}$ & Treatment & Prognosis \\
\hline Present case & 22 & F & Thoracic & Primary & Intralesional resection + CT & NED (18 mo. post op.) \\
Turanli, et al (4) & 65 & F & Lumbar & Primary & Marginal resection & NED (13 mo. post op.) \\
Kirollos, et al (2) & 58 & M & Thoracic & Metastasis & Partial resection + RT & DOD (6 mo. post op.) \\
Ogose, et al $(8)$ & 44 & M & Thoracic & Metastasis & Intralesional resection + RT & DOD (5 mo. post op.) \\
Ogose, et al $(8)$ & 57 & F & Thoracic & Metastasis & Marginal resection & DOD (7 mo. post op.) \\
Lee, et al $(9)$ & 39 & F & Cervical & Metastasis & Resection & NA \\
\hline
\end{tabular}

CT, chemotherapy; RT, radiotherapy; mo., months; op. operation; NED, no evidence of disease; DOD, died of disease; NA, not available.
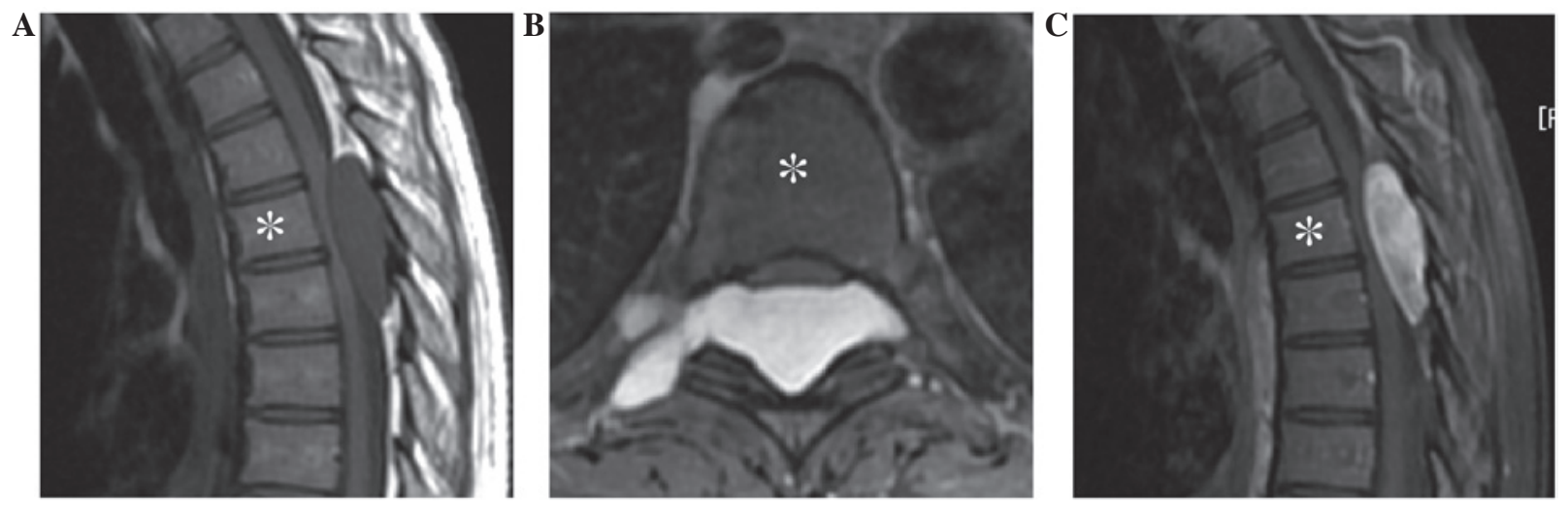

Figure 1. Magnetic resonance imaging of the thoracic spine revealed a dumbbell-shaped, epidural tumor progressing in the right 5 th thoracic vertebrae $(*)$ foramen, exhibiting (A) low intensity on T1-weighted imaging (sagittal view), (B) high intensity on T2-weighted imaging (axial view), and (C) diffuse enhancement on gadolinium-enhanced T1-weighted fat-suppression imaging (sagittal view).
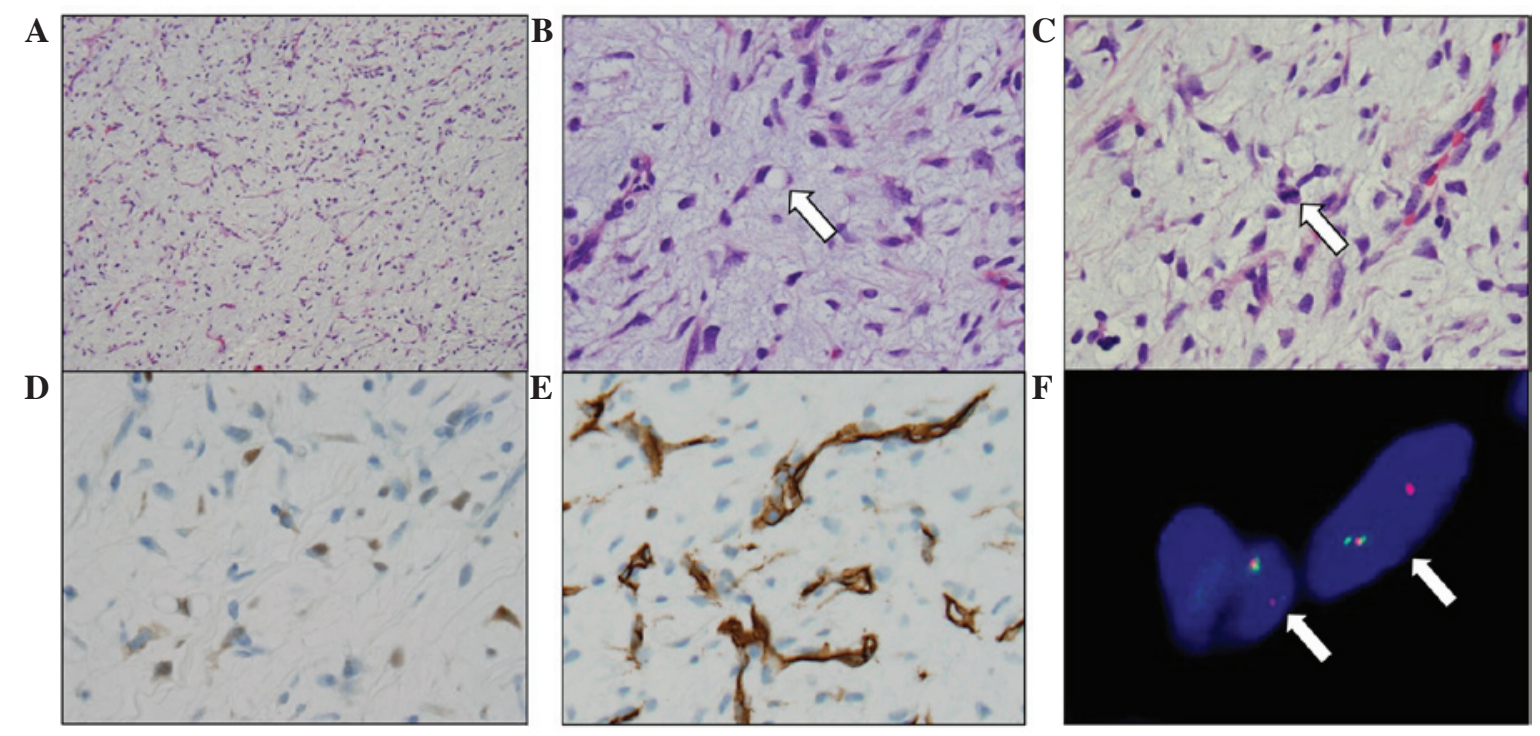

Figure 2. (A) Low-power microscopy revealed proliferation of uniform, mononuclear, short spindle- or oval-shaped cells arranged in a multi-nodular pattern (H\&E; magnification, x40). In the high-power view, (B) lipoblasts and (C) mitosis were scattered (arrows) (H\&E; magnification, x200). (D) Tumor cells were positive for S-100 protein (magnification, x200). (E) A 'chicken-wire' pattern of capillary vasculature was visible (CD34 staining; magnification, x200). (F) Fluorescence in situ hybridization analysis revealed a split signal of the DNA-damage-inducible transcript 3 gene (arrows). H\&E, hematoxylin and eosin.

of capillary vasculature was clearly visible following CD34 staining (Fig. 2E). The MIB1 labeling index was $\sim 6.7 \%$. Furthermore, additional fluorescence in situ hybridization using a break-apart probe for the DNA-damage-inducible transcript 3 gene on $12 \mathrm{q} 13$ revealed a rearrangement (Fig. $2 \mathrm{~F}$ ). Finally, the tumor was diagnosed as an MLS of French 
Federation of Cancer Centers Sarcoma Group grade 1, based on the pathological and genetic findings (7). As additional whole-body ${ }^{18} \mathrm{~F}$-fludeoxyglucose (FDG) positron emission tomography (PET)/CT revealed no other tumor, excluding an ovarian cyst, the epidural tumor of the thoracic spine was diagnosed as primary.

Adjuvant therapy was performed for any possible residual tumor remaining following the intralesional resection. As radiotherapy may cause myelopathy, the patient instead underwent three courses of adjuvant chemotherapy with doxorubicin (60 mg/m²/course) and ifosfamide $\left(7.5 \mathrm{~g} / \mathrm{m}^{2} /\right.$ course $)$, uneventfully. At 6 months after the surgery for the epidural tumor, the patient received an oophorocystectomy, and pathological diagnosis of an endometrial cyst was confirmed. The patient has exhibited no symptoms or signs of local recurrence or metastasis for 18 months postoperatively.

\section{Discussion}

To the best of our knowledge, only five cases of epidural MLS of the spine have been reported previously in the English language literature; one case of primary (4) and four cases of metastatic $(2,8,9)$ (Table I). The present case is the second case of primary epidural MLS of the spine.

As the occurrence of MLS in the epidural space is exceedingly rare and its MRI findings are very similar to those of schwannoma, determining a correct preoperative diagnosis without pathological analysis is considered to be challenging $(10,11)$. MRI of MLS typically shows lacy or linear amorphous high-intensity foci of fat within a predominantly low-intensity mass on T1-WI, intermediate signal intensity foci within a high-intensity mass on T2-WI, and homogeneous enhancement throughout the mass by gadolinium (3). Because of the quantity of fat and myxoid material, the degree of cellularity and vascularity, and the presence of necrosis, MLS may mimic cystic tumors $(12,13)$. Similarly, MRIs of schwannomas usually reveal low to iso intensity on T1-WI, high intensity on T2-WI, and homogeneous or heterogeneous intensity on gadolinium-enhanced T1-weighted fat-suppression imaging (14).

Schwannomas occasionally contain cystic degeneration and, although most $(65.2 \%)$ are intradural extramedullary lesions, $33.0 \%$ and $1.3 \%$ are dumbbell tumors and epidural tumors, respectively (15). Furthermore, among all dumbbell tumors, schwannomas are the most common (69\%), and Eden Type III (extradural and paravertebral type) is the most frequent in both all dumbbell tumors (53\%) and schwannoma (48\%) (16). Thus, from the localization, dumbbell-shaped form (Eden type III) and MRI findings of the present case, the preoperative diagnosis of schwannoma was considered to be reasonable.

FDG-PET/CT has a critical detection limit in relation to small lesions, particularly in tumors that exhibit low ${ }^{18} \mathrm{~F}-\mathrm{FDG}$ uptake, including MLS (17). Therefore, there is the possibility that other small primary and/or metastatic lesions existed in the present patient. However, based on the facts that no other tumor, excluding the endometrial cyst, was revealed on additional radiological analyses, and that the patient has displayed no symptoms or signs of local recurrence or metastasis for 18 months, the tumor was diagnosed as a primary, and not metastatic, epidural MLS of the thoracic spine.
It is clear that wide resection is the standard procedure for soft tissue sarcomas, including MLS $(4,5,13)$. However, in the present case, it was difficult to resect the tumor with an adequate margin due to its location and the compression of the spinal cord causing progressive myelopathy, even if the tumor could have been correctly diagnosed as MLS preoperatively. Although the previously reported case of primary epidural MLS with marginal resection showed no local recurrence (4), adjuvant therapy was considered to be indicated in the current case to prevent further recurrence and metastasis. MLS responds to chemotherapy and radiotherapy $(5,6,18)$; however, adjuvant radiotherapy was not selected in the present case in order to avoid radiation-induced myelopathy. Instead, adjuvant chemotherapy with doxorubicin and ifosfamide was selected.

In conclusion, the current study reports a case of primary epidural, dumbbell-shaped MLS, which radiologically resembled a schwannoma. Although we were unable to resect the tumor with an adequate margin, the patient remains disease-free 18 months after the intralesional tumor resection and subsequent adjuvant chemotherapy. When radiologically diagnosing spinal epidural tumors, clinicians should consider the possibility of MLS as well as schwannoma. Additional treatment with chemotherapy appeared to be useful in the current case of MLS.

\section{Acknowledgements}

This research was supported in part by a Grant-in-Aid for Young Scientists (B) (no. 25861331) from the Japan Society for the Promotion of Science.

\section{References}

1. Antonescu CR and Ladanyi M: Myxoid liposarcoma. In: WHO Classification of Tumours of Soft Tissue and Bone. 4th Edition. Fletcher CDM, Bridge JA, Hogendoorn PCW and Mertens F (eds.). International Agency for Research on Cancer, Lyon, France, pp 39-41, 2013.

2. Kirollos R, Koutsoubelis G, Ross S and Al Sarraj S: An unusual case of spinal metastasis from a liposarcoma. Eur J Surg Oncol 22: 303-305, 1996.

3. Sung MS, Kang HS, Suh JS, Lee JH, Park JM, Kim JY and Lee HG: Myxoid liposarcoma: Appearance at MR imaging with histologic correlation. Radiographics 20: 1007-1019, 2000.

4. Turanli S, Ozer H, Ozyurekoglu T and Cakiroglu E: Liposarcoma in the epidural space. Spine 25: 1733-1735, 2000.

5. Katz D, Boonsirikamchai P, Choi H, Lazar AJ, Wang WL, Xiao L, Park MS, Ravi V, Benjamin RS and Araujo DM: Efficacy of first-line doxorubicin and ifosfamide in myxoid liposarcoma. Clin Sarcoma Res 2: 2, 2012.

6. Chung PW, Deheshi BM, Ferguson PC, Wunder JS, Griffin AM, Catton CN, Bell RS, White LM, Kandel RA and O'Sullivan B: Radiosensitivity translates into excellent local control in extremity myxoid liposarcoma: A comparison with other soft tissue sarcomas. Cancer 115: 3254-3261, 2009.

7. Coindre JM: Grading and staging of sarcomas. In: WHO Classification of Tumours of Soft Tissue and Bone. 4th Edition. Fletcher CDM, Bridge JA, Hogendoorn PCW and Mertens F (eds.) International Agency for Research on Cancer, Lyon, France, pp 17-18, 2013.

8. Ogose A, Hotta T, Inoue Y, Sakata S, Takano R and Yamamura S: Myxoid liposarcoma metastatic to the thoracic epidural space without bone involvement: Report of two cases. Jpn J Clin Oncol 31: 447-449, 2001.

9. Lee SY, Kim HJ, Park SY, Park SH and Chung SK: Myxoid liposarcoma involving the liver, subcutaneous tissue and epidural space in a polycystic disease patient. Clin Nucl Med 33: 507-509, 2008 
10. Kobayashi H, Kotoura Y, Sakahara H, Hosono M, Hosono M, Tsaboyama T, Yamamuro T, Endo $\mathrm{K}$ and Konishi J: Schwannoma of the extremities: Comparison of MRI and pentavalent Technetium-99m-Dimercaptosuccinic acid and Gallium-67-citrate scintigraphy. J Nucl Med 35: 1174-1178, 1994

11. Liu Y, Chen X, Wang T and Wang Z: Imaging observations of a schwannoma of low malignant potential in the anterior abdominal wall: A case report. Oncol Lett 8: 1159-1162, 2014

12. Jelinek JS, Kransdorf MJ, Shmookler BM, Aboulafia AJ and Malawer MM: Liposarcoma of the extremities: MR and CT findings in the histological subtypes. Radiology 186: 455-459, 1993.

13. Sundaram M, Baran G, Merenda G and McDonald DJ: Myxoid liposarcoma: Magnetic resonance imaging appearances with clinical and histological correlation. Skeletal Radiol 19: 359-362, 1990.

14. De Verdelhan O, Haegelen C, Carsin-Nicol B, Riffaud L, Amlashi SF, Brassier G, Carsin M and Morandi X: MR imaging feature of spinal schwannomas and meningiomas. J Neuroradiol 32: 42-49, 2005.
15. Hirano K, Imagama S, Sato K, Kato F, Yukawa Y, Yoshihara H, Kamiya M, Deguchi M, Kanemura T, Matsubara Y, et al: Primary spinal cord tumors: Review of 678 surgically treated patients in Japan. A multicenter study. Eur Spine J 21: 2019-2026, 2012.

16. Ozawa H, Kokubun S, Aizawa T, Hoshikawa T and Kawahara C: Spinal dumbbell tumors: An analysis of a series of 118 cases. J Neurosurg Spine 7: 587-593, 2007.

17. Brenner W, Eary JF, Hwang W, Vernon C and Conrad EU: Risk assessment in liposarcoma patients based on FDG PET imaging. Eur J Nucl Med Mol Imaging 33: 1290-1295, 2006.

18. Guadagnolo BA, Zagars GK, Ballo MT, Patel SR, Lewis VO, Benjamin RS and Pollock RE: Excellent local control rates and distinctive patterns of failure in myxoid liposarcoma treated with conservation surgery and radiotherapy. Int $\mathrm{J}$ Radiat Oncol Biol Phys 70: 760-765, 2008 . 